\title{
Neutrinos and electrons in background matter
}

\author{
Alexander Studenikin \\ Department of Theoretical Physics and Skobeltsyn Institute of Nuclear Physics, Moscow State \\ University, 119992 Moscow, Russia \\ E-mail: studenik@srd.sinp.msu.ru
}

\begin{abstract}
We present a rather powerful method in investigations of different phenomena that can appear when neutrinos and electrons propagate in background matter. This method is based on the use of the modified Dirac equations for particles wave functions, in which the correspondent effective potentials accounting for the matter influence on particles are included.
\end{abstract}

Within the standard model interaction of electron neutrinos and electrons with matter composed of neutrons, the modified Dirac equations are [1, 2, 3, 4]

$$
\left\{i \gamma_{\mu} \partial^{\mu}-\frac{1}{2} \gamma_{\mu}\left(c_{l}+\gamma_{5}\right) \tilde{f}^{\mu}-m_{l}\right\} \Psi^{(l)}(x)=0,
$$

where for the case of neutrinos $m_{l}=m_{\nu}$ and $c_{l}=c_{\nu}=1$, whereas for electrons $m_{l}=m_{e}$ and $c_{l}=c_{e}=1-4 \sin ^{2} \theta_{W}$. For unpolarized matter $\widetilde{f}^{\mu}=\frac{G_{F}}{\sqrt{2}}\left(n_{n}, n_{n} \mathbf{v}\right), n_{n}$ and $\mathbf{v}$ are, respectively, the neutron number density and overage speed. We have obtained the wave functions for neutrinos and electrons in the following form [1, 2, 3, 4]

$$
\Psi_{\varepsilon, \mathbf{p}, s}^{(l)}(\mathbf{r}, t)=\frac{e^{-i\left(E_{\varepsilon}^{(l)} t-\mathbf{p r}\right)}}{2 L^{\frac{3}{2}}}\left(\begin{array}{c}
\sqrt{1+\frac{m_{l}}{E_{\varepsilon}^{(l)}-c \alpha_{n} m_{l}}} \sqrt{1+s \frac{p_{3}}{p}} \\
s \sqrt{1+\frac{m_{l}}{E_{\varepsilon}^{(l)}-c \alpha_{n} m_{l}}} \sqrt{1-s \frac{p_{3}}{p}} e^{i \delta} \\
s \varepsilon \sqrt{1-\frac{m_{l}}{E_{\varepsilon}^{(l)}-c \alpha_{n} m_{l}}} \sqrt{1+s \frac{p_{3}}{p}} \\
\varepsilon \sqrt{1-\frac{m_{l}}{E_{\varepsilon}^{(l)}-c \alpha_{n} m_{l}}} \sqrt{1-s \frac{p_{3}}{p}} e^{i \delta}
\end{array}\right),
$$

where the energy spectra are

$$
E_{\varepsilon}^{(l)}=\varepsilon \sqrt{\mathbf{p}^{2}\left(1-s \alpha_{n} \frac{m}{p}\right)^{2}+m^{2}}+c_{l} \alpha_{n} m_{l}, \quad \alpha_{n}= \pm \frac{1}{2 \sqrt{2}} G_{F} \frac{n_{n}}{m_{l}},
$$

$p, s$ and $\varepsilon$ are particles momenta, helicities and signs of energy, " \pm " corresponds to $e$ and $\nu_{e}$.

The developed approach establishes a basis for investigation of different phenomena which can arise when neutrinos and electrons move in dense media, including those peculiar for astrophysical and cosmological environments. Within this approach we have investigated new types of electromagnetic radiation which can be emitted by neutrinos and electrons moving in dense matter (the "spin light" of neutrino and electron in matter, $S L \nu$ and $S L e$ respectively).

[1] Studenikin A and Ternov A 2005 Phys.Lett. B 608107

[2] Grigoriev A, Studenikin A and Ternov A 2005 Phys.Lett. B 622199

[3] Studenikin A 2006 J.Phys.A: Math. Gen. 396769

[4] A.Grigoriev, S.Shinkevich, A.Studenikin, A.Ternov, I.Trofimov, hep-ph/0611103 